\title{
Driving Forces behind the Development and Stabilization of Knowledge Organization Systems in Digital Environments
}

\author{
Jörgen Jaanus and Tobias Ley \\ Tallinn University, Institute of Informatics, Narva road 25, \\ 10120, Tallinn, Estonia \\ \{Jorgen.jaanus, tobias.ley\} @tlu.ee
}

\begin{abstract}
The emergence of Digital Ecosystems can be endorsed by creating shared conceptualizations. Knowledge Organization Systems (KOS) form a backbone of organizing knowledge. Focusing in developing KOS and having its present and future requirements in mind would eventually support knowledge sharing and learning at collective level. Three types of KOS are distinguished: a) private level KOS; b) arbitrary KOS; c) methodic KOS. Knowledge Maturing can be described as goal-oriented learning on a collective level. In line with the knowledge maturing model, we assume that stabilizing and dynamic forces co-exist in all organizations which seek a dynamic equilibrium between further development and stabilization. Identifying and measuring these forces would help in the effective development of Knowledge Organization Systems and consequently support planning and development of information systems.
\end{abstract}

Keywords: Knowledge Organization Systems, Knowledge Maturing, Digital Ecosystems.

\section{Background Concepts}

Digital Ecosystems support modern networked economies being a next generation information and communications technology for ensuring that information and knowledge are shared across organizations as quickly and effectively as possible [1]. The functional characteristics relate digital ecosystems to achieving cooperation, knowledge sharing, accessing, editing and extrapolating data in order to facilitate learning, knowledge flow and information [2].

Within those ecosystems there are nodes which have their internal development mechanisms which can be described as goal-oriented learning on a collective level defined as knowledge maturing. Knowledge maturing process consists of five consecutive stages: expressing ideas, distributing in communities, formalizing, ad-hoc learning and standardization [3]. Hereby it is claimed that those stages appear in consecutive organizational settings. Ideas are typically expressed in small communities of practice while the standardization can appear at industry-wide level. 
Ontologies are taken as a core enabler for Digital Ecosystems which is built from the entire residing knowledge while Knowledge Organization Systems (KOS) are a development framework for that.

KOS are referred to as levels of semantics [4], maturity of knowledge organization [5], spectrum of knowledge representation [6] or just ontology spectrum [7]. The key difference in those concepts is whether ontologies are considered just formal ontologies whose concepts and relations have definitions that are stated in logic or in some computer-oriented language that can be automatically translated to logic or ontologies are considered on the broader spectrum including both formal and terminological ontologies as distinguished in [8]. There are four knowledge organization systems that can be used to model and organize concepts and to describe terms semantically: controlled vocabularies, taxonomies, thesaurus, and ontologies. [4] Controlled vocabulary is described as a weaker end of this spectrum. Adding structure, hierarchy and child-parent relationships to the controlled vocabulary taxonomy is created. Further from taxonomy thesaurus represent equivalence, homographic, hierarchical, and associative relationships. Using richer semantic relationships among terms and attributes, as well as strict rules about how to specify terms and relationships leads to ontologies. The development of those four KOS in this particular sequence is considered as semantic continuum.

KOS have an important role in each of the knowledge maturing stage. As knowledge matures, more complex structure is needed for knowledge sharing and technical interoperability at the broader scale. The concept of KOS as the core of Digital Ecosystems has shared conceptualization as an essential development mechanism. Private level KOS exist as idiosyncratic and for mainly private use. From this state KOS mature and then gain the guiding role. As an opposite to private level KOS it can be entitled public level KOS which has to distinguishable roles: arbitrary and methodic which roughly correspond to the maturing and guidance in Knowledge Maturing model.

\section{Conceptual Assumptions}

Hereby it is claimed that KOS develop and stabilize to the level which is optimal for knowledge sharing requirements at a given stage of knowledge maturing. Knowledge sharing takes place between individuals in communities, communities in organizations and organizations within cooperation networks. KOS are functioning as boundary objects between those functional units which enable cross-boundary flow of information and knowledge.

Boundary objects are plastic, interpreted differently across communities but with enough immutable content to maintain integrity [9]. The role of the boundary object is not the by-product of organizing knowledge but it is essential to consider KOS as artifacts becoming mediators of distributed cognition as described by Wallace and Ross [10]. This perspective has broadened the value of KOS from solely standardization and findability to coordination and sense-making, consequently fuelling the efforts to advance towards the higher end of semantic spectrum.

The table below depicts the connections between KOS and knowledge maturing stages within consecutive organizational settings. 
Table 1. The congruence between KOS, Knowledge Maturing stages and structural units

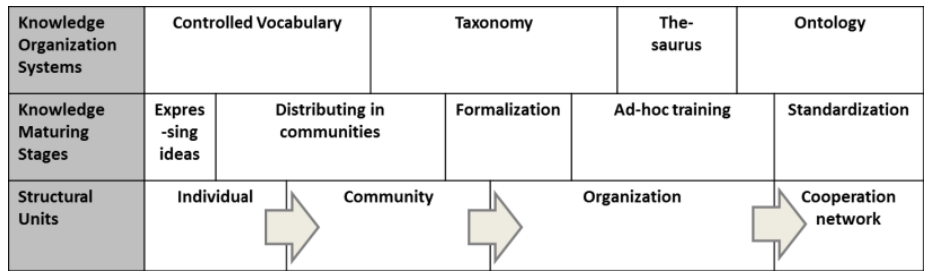

As knowledge maturing leads to ontology development, enterprise application software applications have to be adjusted according to the knowledge maturing requirements which consequently become the cornerstone of long-term competitiveness. For gaining anticipated functionality of any information system it has to incorporate both flexibility and strict standards for information exchange.

As a consequence of ontology development more patterns appear between nodes which makes the overall Digital Ecosystem more dynamic. According to chaos theory in an extremely dynamic organization the boarders and the identity of the organization become open and fluid and cooperative external relationships are sometimes more important than internal ones. If the speed of development is much faster outside the structural unit than inside, the organization moves from equilibrium to the edge of chaos. In this situation a self-organizing ability emerges which is capable of creating a new order [11]. The ability of the organization to adapt to its ecosystem is considerably increased. If the organizations have difficulties finding the right adjustments to their dynamic surroundings the stabilizing forces prevail and KOS have lower interoperability needs. According to Knowledge Maturing model dynamic forces appear through generating new ideas and maturing those ideas throughout the entire ecosystem. Stabilizing forces are observable through organizational guidance. There are dynamic and stabilizing forces which influence the development of KOS and Knowledge Maturing.

Research for identifying and measuring those forces would add focus and precision to the development of KOS and consequently support planning and development of information systems. The existing and anticipated connectivity between various nodes in Digital Ecosystems would be the basis of understanding and measuring those forces.

\section{Research Approach}

Based on this conceptual approach the next step is developing practical model which has Digital Ecosystem as the unit of analysis with three levels: a) an individual belonging to the community; b) community member belonging to the organization; c) knowledge worker as part of industry/ cooperation network.

According to the conceptual assumptions we have to investigate three different types of KOS. First, private level KOS are measured based on respondents' ability to recognize and form categories from pre-given objects. Second, arbitrary KOS is based 
on the questions about work processes depicting regularly appearing situations. Third, methodic KOS are derived from Enterprise Application Software applications.

The analogous research approach has been successfully used by APOSDLE in the work place learning study [12].

\section{Conclusions}

In this paper we have modeled and presented several concepts which provide insight to organizing knowledge. KOS develop and stabilize to the level which is optimal for knowledge sharing requirements at a given stage of knowledge maturing.

There are connections between KOS and knowledge maturing stages within consecutive organizational settings. Two forces coexist which influence development of KOS and Knowledge Maturing. Dynamic forces appear through generating new ideas and maturing those ideas throughout the Digital Ecosystem and stabilizing forces appear through organizational guidance.

The framework of Digital Ecosystems provides context for understanding and measuring those dynamic and stabilizing forces in order to support planning and development of information systems.

\section{References}

1. Price, C., Turnbull, D.: The organizational challenges of global trends. A McKinsey global survey. McKinsey Quarterly (2007)

2. Digital Business Ecosystems, Luxembourg, Office of Official Publications of European Communities (2007)

3. Maier, R., Schmidt, A.: Characterizing Knowledge Maturing. In: 4th Conference Professional Knowledge Management (WM 2007), Potsdam, Germany (2007)

4. Cardoso, J.: The Syntactic and the Semantic Web. Semantic Web Services: Theory, tools and applications. Information Science Reference, New York (2007)

5. Brun, R., Hinkelmann, K., Telesko, R., Thönssen, B.: Towards an Integrated Approach to Assess the Potential of an Enterprise to Mature Knowledge. In: WM 2009, 5th Conference of Professional Knowledge. Management, GI-Edition LNI P-145, pp. 440-444 (2009)

6. Davis, M.: Semantic Wave 2008 Report: Industry Roadmap to Web 3.0 \& Multibillion Dollar Market Opportunities (2008), http: / / www . project 10x.com

7. Lassila, O., McGuinness, D.: The Role of Frame-Based Representation on the Semantic Web (2002)

8. Lacasta, J., Nogueras-Iso, J., Zarazaga-Soria, F.: Terminological Ontologies (2010)

9. Star, S.L., Griesemer, J.R.: Institutional Ecology, 'Translations' and Boundary Objects: Amateurs and Professionals in Berkeley's Museum of Vertebrate Zoology (1989)

10. Wallace, B., Ross, A.: Beyond Human Error: Taxonomies and Safety Science. Ergonomics 51(9) (2006)

11. Caluwe, L., Vermaak, H.: Thinking about Change in Different Colours (2008)

12. De Hoog, R., Kooken, J., Ley, T., Kump, B., Lindstaedt, S.: Advanced Process-Oriented Self-Directed Learning Environment (2008) 